\title{
Potential functions and the characterization of economics-based information
}

\author{
Emmanuel Haven ${ }^{1}$ \\ School of Management; Institute of Finance and IQSCS; University of Leicester, \\ Leicester, United Kingdom
}

\begin{abstract}
The formulation of quantum mechanics as a diffusion process by Nelson [1] provides for an interesting approach on how we may transit from classical mechanics into quantum mechanics. Besides the presence of the real potential function, another type of potential function (often denoted as 'quantum potential') forms an intrinsic part of this theory. In this paper we attempt to show how both types of potential functions can have a use in a resolutely macroscopic context like financial asset pricing. We are particularly interested in uncovering how the 'quantum potential' can add to the economics-based relevant information which is already supplied by the real potential function.
\end{abstract}

Key words: potential functions, information, asset pricing

\section{Introduction}

The stochastic approach to quantum mechanics which dates back from the original work by Nelson [1] shows the emergence of a so called 'quantum potential'. This potential also occurs (then without the use of the rationalized Planck constant, $\hbar$ ) in what Paul and Baschnagel (p.87) [2] term the "hydrodynamic formulation of Newtonian mechanics". The relation between hydrodynamics and quantum mechanics dates back from the work by Madelung [3]. Holland [4] (p. 507) indicates that in "this analogy between quantum mechanics and hydrodynamics....the probability density is proportional to the fluid density and the phase of the wavefunction is a velocity potential." So far, we have not yet mentioned the most well known source from where the quantum potential may be originating from. This is the so called de Broglie-Bohm theory (Bohm [5]; [6]; Bohm and Hiley [7]). We want to make a careful note at

$\overline{1}$ e-mail: eh76@le.ac.uk; phone: 00441162523955 
the very beginning of this paper by again citing Holland [4] who says that one needs (p. 524) "to discriminate ...(the) hydrodynamic interpretation from the de Broglie-Bohm interpretation." And he continues (p. 525) "The de BroglieBohm theory involves a further level of interpretation of quantum mechanics beyond the hydrodynamic interpretation: this includes both the Eulerian and Lagrangian pictures (or any other formulation) and it should not be identified with either." This important point of view is also confirmed by Elbaz [8] (p. 68) who says that in "Nelson's stochastic mechanics .....particles retain their corpuscular character whereas the probability of observing any property...is wave mechanical.... This interpretation separates the corpuscular aspects from the wave mechanical aspects that exist side by side in de Broglie's interpretation."

In this paper we attempt to show how this type of potential can be used (next to the so called real potential) as an aid in modelling information in an asset pricing context. The paper will show some elementary results which, we hope, can indicate a degree of promise for further developments in much more sophisticated asset pricing contexts. This paper continues in the line of some recent research which has attempted to show the usefulness of applying quantum mechanical concepts in macroscopic (for instance social science) domains. We hereby give some examples of this type of research. Khrennikov [9] was one of the very first authors to successfully venture into applying quantum mechanical techniques to social science. In the area of mathematical finance, one of the earliest papers which applies quantum mechanical techniques to financial option pricing theory is by Segal and Segal [10]. In a somewhat related vein, Ishio and Haven [11] attempt to apply the stochastic approach to quantum mechanics in financial option pricing. The quantum potential in the context of asset pricing (and from the perspective of information theory) is discussed in the 'Discussion and Summary' section in a paper by Hawkins and Frieden [12] (see also Haven [13]). Baaquie [14] and [15] analyzes financial theory from a path integral point of view and he thereby gives important insights on how quantum mechanical concepts can find their way in financial asset pricing theory. Baaquie [16] also actively involves the idea of a real potential within a fundamental microeconomis setting. Another paper where potential functions are employed is in Haven and Khrennikov [17] where the concept of tunnelling (quantum-like) is used to describe financial arbitrage. In the area of psychology, work by Busemeyer et al. [18] and Khrennikov and Haven [19] argue how probability interference can explain paradoxical decision making in economics. We also note work in the area of political science where Khrennikova, Haven and Khrennikov [20] introduce open quantum system dynamics to formalize uncertainty resolution of voter's preferences. We could cite much more work. The book by Khrennikov [21] is a great source from which to obtain a solid overview of other recent applications in psychology and economics and other areas. See also Haven and Khrennikov [22]. 
The main goal of this paper consists in showing how we can begin to pragmatically use the real and quantum potentials in an elementary economics (or finance) setting. We hinge our approach for a large part on the work by Nelson [1] because we believe that it is precisely this point of view which shows quite explicitly a gentle transition between classical and quantum mechanics. Nelson's approach however was clearly not developed with social science applications in mind and this paper therefore aims to indeed apply elements of this theory to a non-physics setting.

From the outset, it is quite important to remark that one of the main messages of this paper consists in showing that there may exist concepts from the quantum mechanical formalism which can be employed with some positive use to elucidate better how information (in this paper we mention preferences for risk) could be better modelled with the help of the (real and also quantum) potential. This could imply that the decision making processes (since we discuss preferences, decision makers have towards risk in the sequel of the paper below) could have a quantum mechanical character. This could imply that there maybe a quantum physical brain making such decisions. But this author will not make this claim. We just keep the argument really at the level of: i) there is a formalism from quantum mechanics which can aid in describing an event which relates to modelling information and, ii) if that is the case, there is a wider framework, mainly described by the hydrodynamic approach which can provide for some dynamical predictions. Whether the use of this approach means the decision maker shows features of possessing a quantum physical brain, we do not know.

We start from the 'ground up' so to speak, by first considering the degrees of commutation and uncertainty in a macroscopic setting. We then continue arguing what informational 'advantage' the real potential has over the quantum potential in a simple finance setting. The sequence of forthcoming sections in the paper can be briefly described as follows. In the next section of the paper, we introduce the idea of a commutation equation in social science. In the section following, we introduce degrees of uncertainty. After that section we are then concerned with analyzing how the real and quantum potential functions can aid in modelling time dependent price changes.

\section{A commutation equation in social science?}

From an operator point of view, say if $\widehat{x_{0}}$ is a position operator and $\widehat{p_{0}}$ is a momentum operator, then it is well known from elementary quantum physics that if one can not find an arbitrary well behaved function, $f(x)$ such that $\widehat{x_{0}} \widehat{p_{0}} f(x) \neq \widehat{p_{0}} \widehat{x_{0}} f(x)$ then $\widehat{x_{0}}$ and $\widehat{p_{0}}$ will commute. However, when operators are such that $\widehat{x_{0}}, \widehat{p_{0}} \in \mathbb{R}$ then $\widehat{x_{0}} \widehat{p_{0}} f(x)-\widehat{p_{0}} \widehat{x_{0}} f(x)=0$. Now consider: 


$$
\widehat{x_{p} \widehat{p_{0}}}-\widehat{p_{p}} \widehat{x_{p}} \neq 0
$$

where $\widehat{x_{p}} ; \widehat{p_{0}} ; \widehat{p_{p}} \in \mathbb{R}$. Equation (1) above, introduces a degree of commutation which is different from the level of commutation obtained when $\widehat{x_{0}} \widehat{p_{0}} f(x)-$ $\widehat{p_{0}} \widehat{x_{0}} f(x) \neq 0$ with $\widehat{p_{0}} \notin \mathbb{R}$, as is the case in quantum mechanics with the Heisenberg Uncertainty Principle. Following Baaquie [14] (p. 99), we can define a similar type of commutation equation as (1) above:

$$
E\left[x_{n}\left(\frac{x_{n+1}-x_{n}}{\epsilon}\right)-\left(\frac{x_{n}-x_{n-1}}{\epsilon}\right) x_{n}\right] \equiv E(C) ;
$$

where $E[$.$] is an expectation operator, and say x_{n}$ is the price of a financial asset at time $n$ and $C$ is some constant. If time is discretized, one can write that $t=n \epsilon$, where $\epsilon$ can for instance be expressed in terms of remaining time (i.e. for instance time needed until an asset matures) (see Baaquie [14] (p. 99)). We remark that in equation (2) above, we could write that $\widehat{p_{0}}=\frac{x_{n+1}-x_{n}}{\epsilon}$ and $\widehat{p_{p}}=\frac{x_{n}-x_{n-1}}{\epsilon}$ and $\widehat{p_{p}}, \widehat{p_{0}} \in \mathbb{R}$. In some sense, equation (2) introduces the idea of so called 'forward and backward mean time derivatives', as they are defined in Nelson's theory [1]. In this paper we follow Paul and Baschnagel's [2] rendering of Nelson's theory. We also adopt the same notation. We henceforth, where appropriate, continue referring to Nelson and Paul and Baschnagel. Let us assume that $\epsilon \rightarrow 0$ then we can write: $\frac{d x_{+}}{d t} \simeq \frac{x_{n+1}-x_{n}}{\epsilon}$ and $\frac{d x_{-}}{d t} \simeq \frac{x_{n}-x_{n-1}}{\epsilon}$. If we assume, as in Nelson [1] and Paul and Baschnagel [2], a Brownian motion of the following type:

$$
d x(t)=b_{+}(x, t) d t+\sigma d W(t) ;
$$

where $d W(t)$ is a Wiener process; $\sigma$ is the diffusion coefficient (see section 2 below); $b_{+}(x, t)$ is the drift function. Since $E(d W(t))=0$, using the notation in equation (2):

$$
D_{+} x(t)=\lim _{\epsilon \rightarrow 0} E\left[\frac{x_{n+1}-x_{n}}{\epsilon}\right]=b_{+}(x, t) .
$$

Similarly, we assume a Brownian motion of the type (Nelson [1] (Paul and Baschnagel [2])):

$$
d x(t)=b_{-}(x, t) d t+\sigma d W(t) .
$$

Using again the notation in equation (2), we write:

$$
D_{-} x(t)=\lim _{\epsilon \rightarrow 0} E\left[\frac{x_{n}-x_{n-1}}{\epsilon}\right]=b_{-}(x, t) .
$$




\section{A commutation equation in social science: degrees of uncertainty}

As in Baaquie [14], let us define $E(C)$ in equation (2) to be equal to $\sigma^{2}$. Let us now use equation (2) to distinguish different levels of uncertainty. We propose the following sequence of levels of uncertainty. We repeat equation (2) again and we now add the squared volatility term:

$$
E\left[x_{n}\left(\frac{x_{n+1}-x_{n}}{\epsilon}\right)-\left(\frac{x_{n}-x_{n-1}}{\epsilon}\right) x_{n}\right] \equiv \sigma^{2} .
$$

\subsection{Varying non-zero levels of uncertainty}

If we fix, $x_{n}$, then:

$$
x_{n} E\left[\left(\frac{x_{n+1}-x_{n}}{\epsilon}\right)-\left(\frac{x_{n}-x_{n-1}}{\epsilon}\right)\right] \equiv \sigma_{*}^{2}<\sigma^{2} .
$$

We need to make several remarks relative to equations (7) and (8).

- if in the discretization of time, $t$, we do not express $\epsilon$ in terms of remaining time, then equations (7) and (8) can be written without a minus sign (before the expectation operator). This is surely not always the case. In the context of option pricing, Baaquie [14], shows that the left hand side of equation (7) would need a minus sign (before the expectation operator) because the time derivatives take on negative signs (i.e. via the use of $T-t=n \epsilon$, where $T$ is the maturity time of an option)

- inspection of equation (8) also immediately indicates a substantial departure from the core interpretation of the Heisenberg uncertainty principle. One of the obvious reasons consists in arguing that if one were to attribute to $x_{n}$ a specific value, then infinite uncertainty on the momentum measurement would need to ensue. This is clearly not the case here.

We can write the inequality $\sigma_{*}^{2}<\sigma^{2}$ in equation (8), since a level of uncertainty has now been removed by the mere fact that $x_{n}$ is known. We can possibly further refine the level of uncertainty by fixing, $x_{n}$, and setting $\frac{d x_{+}}{d t} \simeq \frac{x_{n+1}-x_{n}}{\epsilon}$ and $\frac{d x_{-}}{d t} \simeq \frac{x_{n}-x_{n-1}}{\epsilon}$. We can then write:

$$
x_{n} E\left[\frac{d x_{+}}{d t}-\frac{d x_{-}}{d t}\right] \equiv \sigma_{* *}^{2}<\sigma_{*}^{2}<\sigma^{2} ;
$$

where $\sigma_{* *}^{2}$ reflects the higher degree of precision given the use of continuous derivatives. 
Finally, another additional refinement of the level of uncertainty could be proposed by introducing so called $q$ derivatives which form the backbone of so called 'quantum calculus' (see Kac and Cheung [23]). For the purposes of this paper, we limit ourselves to the use of the basic definitions. For an arbitrary function $f(x)$, the $q$-differential is: $d_{q} f(x)=f(q x)-f(x)$. The $q$-derivative of a function $f(x)$ is: $D_{q} f(x)=\frac{d_{q} f(x)}{d_{q}(x)}=\frac{f(q x)-f(x)}{q x-x}$, and clearly when $q \rightarrow 1$, one obtains the ordinary continuous derivative. As an example, for a function $f(x)=x^{n}, D_{q} f(x)=\frac{x^{n}\left(q^{n}-1\right)}{x(q-1)}$ and $\frac{q^{n}-1}{q-1}=q^{n-1}+\ldots+1=[n]$. We note that $D_{q} f(x)$ can also be formulated with reference to a time scale (see Hilger [24] and Grow and Sanyal [25]). We do not expand on this here. For more information on using $q$-derivatives in a social science setting, see Haven [26].

We can now formulate equation (9) with $q$ derivatives. Thus, for $q \rightarrow 1$, $x_{n} E\left[\frac{d_{q} x_{+}}{d t}-\frac{d_{q} x_{-}}{d t}\right]=\sigma_{* *}^{2}$, but for $q \nrightarrow 1, x_{n} E\left[\frac{d_{q} x_{+}}{d t}-\frac{d_{q} x_{-}}{d t}\right]=\sigma_{* * *}^{2}>\sigma_{* *}^{2}$. We assume that $q$ proxies for some measure of precision of measurement.

\section{Real and quantum potential functions and links with time de- pendent price changes}

Although the real potential function is a very well known object in physics, the 'quantum potential' may be less well known. This section of the paper has as goal to connect both types of potentials to economics and finance. This is still a very novel area of research, even though there exist already a lot of statistical physics applications in social science. Khrennikov [21] was amongst the first to develop an economics understanding of such functions. We want to argue in this section of the paper that both those functions can have an important pragmatic use in social science.

\subsection{The real potential and its links with time dependent price change}

In the theory by Nelson [1] (Paul and Baschnagel[2]) when using the FokkerPlanck partial differential equation (forward and backward), the case when the force on the real potential is the drift function (either $b_{+}(x, t)$ or $b_{-}(x, t)$ ), yields:

$$
-\nabla V=b_{+}(x, t)
$$

We find this drift function back in equation (3). Similarly for $b_{-}(x, t)$, which we can find back in equation (5).

The connection with economics of the negative of the force, $-\nabla V$, is now 
quite straightforward. Depending on the precise definition of the drift term, it can be interpreted as either the expected return on an asset 's price or the time dependent change of the price. The argument that the drift function can be associated with for instance the expected return is not new at all. This argument was already made almost 40 years ago in the basic formulation of the Black-Scholes [27] option pricing theory. However, what is new here is the explicit association of the role of the real potential function with the expected return (or the time dependent change in the price). The precise pinpointing of the level of $b(x, t)$ is dependent on the preferences for risk of decision makers. In economics, such preferences for risk are modelled by the aid of a so called 'utility function'. The 'appropriate' utility function is notoriously hard to determine for any economics problem (and so it would be in the case of the use of real potential functions). Typically, the utility function's degree of concavity (or convexity) is an indicator of risk aversion (or risk preference). Ma [28] is an excellent source for more background on the use of utility functions in an asset pricing context. However, it needs explicit stressing, that in comparison with the utility function, the real potential function is embedded in a much richer, and usable, structure. As an example, within the setting of the Fokker-Planck PDE, if the drift function were to be exactly known, then the time dependent evolution of the probability density function could possibly be described in precise terms: the density function would indicate the likely position of particles in the real potential. Therefore, in an economics context, such density function could indicate the likely position of an asset price. Baaquie [16] provides for a very interesting interpretation when he defines the real potential as the sum of a demand and supply function (of goods). Upon minimizing the real potential (towards price), he obtains an equilibrium price.

\subsection{The 'quantum' potential in quantum mechanics and in Nelsonian me- chanics}

Nelson's theory [1] (Paul and Baschnagel [2]) shows there exists a very gentle transition between classical mechanics and quantum mechanics. We very much hope to be able to clarify in the sequel why this peculiar transition can be useful in a social science setting. Holland [29] shows that if we insert the quantum mechanical wave function of the form $\Psi(x, t)=e^{R(x, t)} e^{\frac{i}{\hbar} S(x, t)}$, where $R(x, t)$ is the amplitude function of the wave function and $S(x, t)$ is the phase of the wave function, into the Schrödinger equation,

$$
i \hbar \frac{\partial \Psi}{\partial t}+\frac{\hbar^{2}}{2 m} \Delta \Psi=V \Psi
$$

then one obtains for the real part: 


$$
\frac{\partial S}{\partial t}+V+\frac{1}{2 m}(\nabla S)^{2}-\frac{\hbar^{2}}{2 m}\left[(\nabla R)^{2}+\Delta R\right]=0
$$

It maybe less well known that the above equation can be obtained, almost identically, in a non-quantum context, when considering Nelson's stochastic approach. Define the probability density function as: $f(x, t)=e^{2 R(x, t)}$, where $R(x, t)$, as per Paul and Baschnagel [2], is now called a scalar field. If one just sets $R^{\prime}=e^{R}$ and executes $\nabla^{2} R^{\prime}$, then we obtain:

$$
\nabla^{2} R^{\prime}=e^{R}\left(\nabla^{2} R+(\nabla R)^{2}\right)=e^{R}\left(\Delta R+(\nabla R)^{2}\right)
$$

or also:

$$
\frac{\nabla^{2} R^{\prime}}{R^{\prime}}=\Delta R+(\nabla R)^{2}
$$

In fact, in the non-quantum context, using the stochastics as proposed in equations (3) and (5), Nelson [1] (Paul and Baschnagel [2]) finds:

$$
\frac{\partial S}{\partial t}+V+\frac{1}{2 m}(\nabla S)^{2}-\frac{m \sigma^{4}}{2}\left[(\nabla R)^{2}+\Delta R\right]=0
$$

Note the obvious absence of the Planck constant. The term $\frac{\nabla^{2} R^{\prime}}{R^{\prime}}$ from equation (15) is an essential part of the so called 'quantum potential', $Q$. Such $Q$ is defined as $Q=\frac{-\hbar^{2}}{2 m} \frac{\nabla^{2} R^{\prime}}{R^{\prime}}$. We remark that this quantum potential also figures in the Newtonian equation as follows: $m a=-\nabla(V+Q)$ (see Holland [29] (p.74)). The right hand side of this equation indicates the 'force' which is derived not only from the real potential, $V$ but also from the quantum potential $Q$. As we already remarked in the introduction to this paper Elbaz [8] for instance, indicates the clear separation of corpuscular from wave mechanical aspects in Nelson's theory. This can be seen very clearly here: $R(x, t)$ is called a scalar field in the classical mechanics context, while it is the amplitude function in the quantum context.

\subsection{Social science based 'non-commuting' and 'commuting' in Newtonian and Nelsonian mechanics}

Nelson [1] (Paul and Baschnagel [2]) defines two velocity fields, $v(x, t)$ and $u(x, t)$, which are respectively, depicting the mean and osmotic velocities. They are defined as: 


$$
\begin{aligned}
& v(x, t)=\frac{1}{2}\left(b_{+}(x, t)+b_{-}(x, t)\right), \\
& u(x, t)=\frac{1}{2}\left(b_{+}(x, t)-b_{-}(x, t)\right) .
\end{aligned}
$$

In Newtonian mechanics $b_{+}(x, t)=b_{-}(x, t)$, and hence $u(x, t)=0$. Note also that if Newtonian mechanics is used, our proposed equation (9) will now become:

$$
x_{n} E\left[\frac{d x_{+}}{d t}-\frac{d x_{-}}{d t}\right] \equiv 0 .
$$

Hence, if we consider our proposed equation (7), there does not exist any level of commutation. This is an intuitive result. In Nelson [1] (Paul and Baschnagel $[2]), u(x, t)$ is also written as:

$$
u(x, t)=\frac{\sigma^{2}}{2} \nabla \ln f
$$

and, using $f(x, t)=e^{2 R(x, t)}$, we can write this slightly differently as $\frac{\sigma^{2}}{2} \nabla \ln e^{2 R(x, t)}=$ $\sigma^{2} \nabla R(x, t)$. Recall the level of uncertainty as proposed in equation (9) and using equations (4), (6) and (19), we get:

$$
x_{n} E\left[\frac{d x_{+}}{d t}-\frac{d x_{-}}{d t}\right]=2 x_{n} u(x, t)=2 x_{n} \sigma^{2} \nabla R(x, t) .
$$

Hence, the uncertainty component, $E\left[\frac{d x_{+}}{d t}-\frac{d x_{-}}{d t}\right]=2 \sigma^{2} \nabla R(x, t)$. The level of uncertainty (as per equation (7) and as seen here in the above equation) is now very explicitly tied to i) $\sigma^{2}$ but also ii) to the gradient of the scalar field (or the amplitude function), $R(x, t)$. In Newtonian mechanics, either $\sigma^{2}=0$ and/or $\nabla R(x, t)=0$ (here $R(x, t)$ is the scalar field). We can observe (see next section) that $\sigma^{2}=0$ would make the $\nabla R(x, t)$ undefinable. See equation $(22)$ below. Recall that the quantum potential: $Q=\frac{-\hbar^{2}}{2 m} \frac{\nabla^{2} R^{\prime}}{R^{\prime}}=\frac{-\hbar^{2}}{2 m}\left[(\nabla R)^{2}+\Delta R\right]$ and using $\sigma^{2}=\frac{\hbar}{m}$ (as in Nelson [1] (Paul and Baschnagel [2])), we can see that the uncertainty in (21) is indeed related to the existence of this 'quantum potential'.

Finally, let us write (21) as:

$$
\nabla R(x, t)=\frac{E\left[\frac{d x_{+}}{d t}-\frac{d x_{-}}{d t}\right]}{2 \sigma^{2}}
$$


which requires $\sigma^{2}$ to be non-zero for $\nabla R(x, t)$ to exist.

\subsection{A first appraisal of the usefulness of the quantum potential}

We recall that $Q=\frac{-\hbar^{2}}{2 m} \frac{\nabla^{2} R^{\prime}}{R^{\prime}}=\frac{-\hbar^{2}}{2 m}\left[(\nabla R)^{2}+\Delta R\right]$. Let us concentrate on $\left[(\nabla R)^{2}+\Delta R\right]$, which can be written as:

$$
\frac{\nabla^{2} R^{\prime}}{R^{\prime}}=\Delta R+\left(\frac{u(x, t)}{\sigma^{2}}\right)^{2} ;
$$

and using (17) this can be re-written as:

$$
\frac{\nabla^{2} R^{\prime}}{R^{\prime}}=\Delta R+\frac{1}{4 \sigma^{4}}\left[b_{+}(x, t)^{2}-2 b_{+}(x, t) b_{-}(x, t)+b_{-}(x, t)^{2}\right] .
$$

Since, $\Delta R=\frac{\partial^{2} R}{\partial x^{2}}$ and since $u(x, t)=\sigma^{2} \frac{\partial R}{\partial x}$ (see Nelson [1]; Paul and Baschnagel [2]) then, if $\sigma$ is a constant: $\Delta R=\frac{1}{\sigma^{2}} \frac{\partial u(x, t)}{\partial x}$. Hence, (23) can be re-written as:

$$
\frac{\nabla^{2} R^{\prime}}{R^{\prime}}=\frac{1}{\sigma^{2}} \frac{\partial u(x, t)}{\partial x}+\frac{1}{4 \sigma^{4}}\left[b_{+}(x, t)^{2}-2 b_{+}(x, t) b_{-}(x, t)+b_{-}(x, t)^{2}\right] .
$$

And this can of course be written out in full as:

$$
\frac{\nabla^{2} R^{\prime}}{R^{\prime}}=\frac{1}{\sigma^{2}} \frac{\partial}{\partial x}\left[\frac{1}{2}\left(b_{+}(x, t)-b_{-}(x, t)\right)\right]+\frac{1}{4 \sigma^{4}}\left[b_{+}(x, t)^{2}-2 b_{+}(x, t) b_{-}(x, t)+b_{-}(x, t)^{2}\right] .
$$

Let us for instance set $b_{+}(x, t)=b$ and $b_{-}(x, t)=c$ where $b \neq c ; b, c \in \mathbb{R} . b$, $c$ can be seen as the time dependent price change of a financial asset. We can now write:

$$
\frac{\nabla^{2} R^{\prime}}{R^{\prime}}=\frac{1}{4 \sigma^{4}}\left[b^{2}-2 b c+c^{2}\right]=\frac{1}{4 \sigma^{4}}(b-c)^{2} .
$$

If we thus set $b_{+}(x, t)=b$ and $b_{-}(x, t)=c$ where $b \neq c ; b, c \in \mathbb{R}$ then from (22), we can re-write (26) as: 


$$
\frac{\nabla^{2} R^{\prime}}{R^{\prime}}=\left(\frac{u(x, t)}{\sigma^{2}}\right)^{2}
$$

We can use $(22)$ or in a more specific situation (i.e. with $b_{+}(x, t)=b$ and $b_{-}(x, t)=c$ where $b \neq c$ and $\left.b, c \in \mathbb{R}\right)(27)$, to write:

$$
u(x, t)=\sigma^{2} \sqrt{\frac{\nabla^{2} R^{\prime}}{R^{\prime}}} .
$$

The level of the time price change, as captured by either $b$ or $c$, is from an economics point of view depending on the decision maker's attitudes towards risk. Such dependence is often modelled in a financial asset pricing context with the help of so called utility (or also preference) functions. See for instance, Ma [28]. Clearly, in (25) we have thus embedded attitudes towards risk. Therefore, $\frac{\nabla^{2} R^{\prime}}{R^{\prime}}$ would have to reflect a preference function towards risk.

Let us recall (20): $x_{n} E\left[\frac{d x_{+}}{d t}-\frac{d x_{-}}{d t}\right]=2 x_{n} u(x, t)$, and if we use (28), then we can express the uncertainty (for $x_{n}$ fixed) as:

$$
x_{n} E\left[\frac{d x_{+}}{d t}-\frac{d x_{-}}{d t}\right]=2 x_{n} \sigma^{2} \sqrt{\frac{\nabla^{2} R^{\prime}}{R^{\prime}}} .
$$

The above equation seems to indicate that with $x_{n}$ fixed and $b_{+}(x, t)=b$ and $b_{-}(x, t)=c$ where $b \neq c$ and $b, c \in \mathbb{R}$, the uncertainty is now function of $\sigma^{2}$ and the term $\frac{\nabla^{2} R^{\prime}}{R^{\prime}}$. For the full classical mechanics equivalent of the quantum potential one need to multiply $\frac{\nabla^{2} R^{\prime}}{R^{\prime}}$ with the factor $\frac{-m \sigma^{4}}{2}$. Then $(26)$, would simply become:

$$
\frac{-m \sigma^{4}}{2} \frac{\nabla^{2} R^{\prime}}{R^{\prime}}=\frac{-m}{8}(b-c)^{2} .
$$

Note also that the probability density function, on the basis of $e^{2 R(x, t)}$, would in this case be very straightforward to calculate. Using (21) and the assumption that for instance $b_{+}(x, t)=b x$ and $b_{-}(x, t)=0$, then employing (21) will yield: $\nabla R=\frac{b x}{2 \sigma^{2}}$. Therefore, $R=\int \frac{b x}{2 \sigma^{2}} d x=\frac{b x^{2}}{4 \sigma^{2}}+$ constant. The probability density function on the basis of $e^{2 R(x, t)}$ (thus as the result of complex conjugation of the polar form of the wave function (or as a given probability density form with a scalar field $R(x, t))$, would then be $f(x)=\exp \left(\frac{b x^{2}}{2 \sigma^{2}}+\right.$ constant $)=a \exp \left(\frac{b x^{2}}{2 \sigma^{2}}\right)$, which is akin to a Normal probability density function with mean zero.

From the development so far, we can remark that not only the real potential 
has a link to preferences for risk, but so does the quantum potential. We have seen this very clearly in (24) above. Remark that the quantum potential does intimately link up with the amplitude function $R(x, t)$. In Haven [31] an argument is made how the wave function, as an information function device, can be connected to economics based (non-observable) state prices, $s$, by using the simple relation: $s=\frac{1}{1+r} \int \exp (2 R(x, t)) d x$, where $r$ is the risk free rate of interest (which is devoid of any preference for risk). Such state prices, $s$, are again reflective of preferences for risk since they can be interpreted as prices paid to obtain a certain price in a state of nature (like the state of the economy for instance).

The existence of the scalar field $R(x, t)$ (or the amplitude function) in fact has a close connection to preferences for risk. The dynamic character of such preferences would clearly be linked to some Hamiltonian function, which then would also include a kinetic energy function. However, we would like to stress the close connection of the potential function terms in that Hamiltonian with the preferences for risk.

\subsection{Which potential can be of most use in social science? A brief discussion.}

An important point can be raised as to the 'quality' of information that the real potential $V$ has, as opposed to quantum potential $Q$. The $\nabla V$ does refer to the drift function of the Brownian motion. In effect, this drift function does incorporate an expected return (or the time dependent price change). However, we do not know what the level is of this expected return (or the time dependent price change). Some 'holy grail' utility function which can aggregate preferences for risk is able to determine this level of expected return (or time dependent price change). It is therefore important to indicate that $\nabla V$ is in fact quite further removed from a utility function than one thinks! In fact $\nabla V$ is only related to the result of such utility function, but we are completely ignorant about the real functional of the utility function. Oppose now to this argument, the state price $s=\frac{1}{1+r} \int \exp (2 R(x, t)) d x$. On prima facie, this price is very closely linked to preferences for risk since such state price is the price paid to guarantee a certain outcome to occur when a state of nature reveals itself. Within the setting of the non-arbitrage theorem (see f.i. Neftci [32]), the positive state prices also solve a system of equations to guarantee non-arbitrage. Hence, under non-arbitrage, precise values of $s$ can be found and precision can be imposed on the amplitude function $R(x, t)$, as long as one can impose bounds on the integral! Hence, there is thus uncertainty: either $R(x, t)$ is known but the integral bounds are unknown or vice versa. However, the argument remains that the $R(x, t)$ function is closely linked to preferences for risk because of the interpretation of the state price. However, in conclusion, the final argument is virtually the same as with $\nabla V$ : to know $R$ 
we would need to know $b_{+}(x, t)$ or $b_{-}(x, t)$ and those functions hide a utility functional! Consider the following train of thought: find $R$ from integrating: $\nabla R(x, t)=\frac{E\left[\frac{d x_{+}}{d t}-\frac{d x_{-}}{d t}\right]}{2 \sigma^{2}}$, but this assumes you need to know the value of the time dependent price change in either $\frac{d x_{+}}{d t}$ or $\frac{d x_{-}}{d t}$. If you were to know this, then $s=\frac{1}{1+r} \int \exp (2 R(x, t)) d x$ can be calculated if the bounds of the integral can be determined if you know the state price (which guarantees no arbitrage asset pricing). There is no unique way to go ahead with finding any of the parameters.

In conclusion, $\nabla V$ and $\nabla Q$ (via $R$ ) give in effect very similar information. However, before we go to finally conclude this, let us just oppose: $\nabla V$ which is say $b_{+}(x, t)$ or $b_{-}(x, t)$ with $\frac{\nabla^{2} R^{\prime}}{R^{\prime}}=\Delta R+\frac{1}{4 \sigma^{4}}\left[b_{+}(x, t)^{2}-2 b_{+}(x, t) b_{-}(x, t)+b_{-}(x, t)^{2}\right]$. If indeed the functionals $b_{+}(x, t)$ or $b_{-}(x, t)$ are not constant and of higher order in $x$ then the force of the quantum potential seems to add more information. But it does not add more information in terms of finding preferences for risk.

We remark also the following. Equation (22) clearly expresses the quantum potential as being very much a function of $\sigma^{2}$. The quantum potential is thus dependent on the diffusion coefficient. This is not the case of the real potential. The real potential refers to the drift terms in the stochastic differential equation (thus not the diffusion terms). Thus in terms of 'added' information, the quantum potential incorporates also diffusivity.

Finally, note also that we could consider an interesting measure by using the ratio: $\frac{\nabla V}{\nabla R}=\frac{\frac{\partial V}{\partial x}}{\frac{\partial R}{\partial x}}=\frac{\partial V}{\partial R}$. Using, $(21)$ and setting $E\left[\frac{d x_{+}}{d t}-\frac{d x_{-}}{d t}\right]=b_{+}(x, t)-$ $b_{-}(x, t)$ and assuming that $\nabla V$ is say $b_{+}(x, t)$ or $b_{-}(x, t)$ (or some other drift altogether), then $\frac{\nabla V}{\nabla R}=\frac{2 b_{+}(x, t) \sigma^{2}}{b_{+}(x, t)-b_{-}(x, t)}$ and if for instance, we set $b_{-}(x, t)=0$ then: $\frac{\nabla V}{\nabla R}=\frac{2 b_{+}(x, t) \sigma^{2}}{b_{+}(x, t)}=2 \sigma^{2}$. This means thus that the measure of the change in the amplitude (or the scalar field) which induces a change in the real potential, will be measured by $\sigma^{2}$. If $\sigma^{2}$ is zero, i.e. if there is no stochasticity, then the impact of the change of the amplitude function on the change in the real potential is zero. In other words, the amplitude function (which is a major input in the wave function and which is a determinant in the probability density function) has no relation at all to the real potential function in that very case.

\section{Acknowledgements}

The author gratefully acknowledges the study leave support received from the University of Leicester. 


\section{References}

[1] Nelson, E.: Derivation of the Schrödinger equation from Newtonian mechanics. Phys. Rev. 150, 1079-1085 (1966)

[2] Paul, W., Baschnagel, J.: Stochastic Processes: From Physics to Finance. Springer Verlag (1999)

[3] Madelung, E.: Quantentheorie in Hydrodynamischer Form. Z. Phys. 40, 322 (1926)

[4] Holland, P.: Computing the wave function from trajectories: particle and wave pictures in quantum mechanics and their relation. Ann. Phys-New York 315, 505-531 (2005)

[5] Bohm, D.: A suggested interpretation of the quantum theory in terms of hidden variables. Phys. Rev. 85, 166-179 (1952a)

[6] Bohm, D.: A suggested interpretation of the quantum theory in terms of hidden variables. Phys. Rev. 85, 180-193 (1952b)

[7] Bohm, D., Hiley, B.: The Undivided Universe: an Ontological Interpretation of Quantum Theory. Routledge.

[8] Elbaz, E.: The Quantum Theory of Particles, Fields and Cosmology. Springer Verlag (1998)

[9] Khrennikov, A. Yu.: Classical and quantum mechanics on information spaces with applications to cognitive, psychological, social and anomalous phenomena. Found. Phys. 29, 1065-1098 (1999)

[10] Segal, W., Segal, I.E.: The Black-Scholes pricing formula in the quantum context. Proc. Natl. Acad. Sci. USA 95, 4072-4075 (1998)

[11] Ishio, H., Haven, E.: Information in asset pricing: a wave function approach. Ann. Phys. (Berlin) 18(1), 33-44 (2009)

[12] Hawkins, R.J., Frieden, B. R.: Asymmetric information and quantization in financial economics. Internat. J. Math. Math. Sci., 2012, doi: $10.1155 / 2012 / 470293(2012)$

[13] Haven, E.: Financial payoff functions and potentials. Lect. Notes Comput. Sc. 8951, 189-195 (2015)

[14] Baaquie, B.: Quantum Finance: Path Integrals and Hamiltonians for Options and Interest Rates. Cambridge University Press (2007)

[15] Baaquie, B.: Interest rates in quantum finance: the Wilson expansion. Phys. Rev. E 80, 046119 (2009)

[16] Baaquie, B.: Statistical microeconomics. Physica A 392(19), 4400-4416 (2013) 
[17] Haven, E., Khrennikov, A.: Quantum-like tunnelling and levels of arbitrage. Int. J. Theor. Phys. 52(11), 4083-4099 (2013)

[18] Busemeyer, J., Wang, Z., Townsend, J.T.: Quantum dynamics of human decision making. J. Math. Psychol. 50, 220-241 (2006)

[19] Khrennikov, A. , Haven, E.: Quantum mechanics and violations of the surething principle: the use of probability interference and other concepts. J. Math. Psychol. 53, 378-388 (2009)

[20] Khrennikova, P., Haven, E., Khrennikov, A. : An application of the theory of open quantum systems to model the dynamics of party governance in the US political system. Int. J. Theor. Phys. 53, 1346-1360 (2014)

[21] Khrennikov, A.: Ubiquitous Quantum Structure: from Psychology to Finance. Springer Verlag (2010)

[22] Haven, E., Khrennikov, A.: Quantum Social Science. Cambridge University Press (2013)

[23] Kac, V., Cheung, P.: Quantum Calculus. Springer Verlag (2000)

[24] Hilger, S.: Analysis on measure chains - a unified approach to continuous and discrete calculus. Results Math. 18, 18-56 (1990)

[25] Grow, D., Sanyal, S.: Brownian motion indexed by a time scale. Stoch. Anal. Appl. 29(3), 457-472 (2011)

[26] Haven, D.: Itô's Lemma with quantum calculus: some implications. Found. Phys. 41(3), 529-537 (2010)

[27] Black, F., Scholes, M.: The pricing of options and corporate liabilities. J. Polit. Econ. 81, 637-659 (1973)

[28] Ma, C.: Advanced Asset Pricing Theory. Imperial College Press (2010)

[29] Holland, P.: The Quantum Theory of Motion. Cambridge University Press (2004)

[30] Itô, K.: On stochastic differential equations. Am. Math. Soc. 4, 1-51 (1951)

[31] Haven, E.: The variation of financial arbitrage via the use of an information wave function. Int. J. Theor. Phys. 47, 193-199 (2008)

[32] Neftci, S.: An Introduction to the Mathematics of Financial Derivatives. Academic Press (2000) 